\title{
Absence of glaucoma in DBA/2J mice homozygous for wild-type versions of Gpnmb and Tyrpl
}

Gareth R Howell ${ }^{1}$, Richard T Libby ${ }^{1,6}$, Jeffrey K Marchant ${ }^{2}$, Lawriston A Wilson ${ }^{1,3}$, Ioan M Cosma1, Richard S Smith ${ }^{1,3}$, Michael G Anderson ${ }^{4}$ and Simon WM John*1,3,5

\begin{abstract}
Address: ${ }^{1}$ The Jackson Laboratory, 600 Main Street, Bar Harbor, Maine, USA, ${ }^{2}$ Department of Anatomy and Cell Biology, Tufts University of Medicine, Boston, MA, USA, ${ }^{3}$ The Howard Hughes Medical Institute, Bar Harbor, Maine, USA, ${ }^{4}$ Department of Physiology and Biophysics, The University of Iowa, Iowa City, IA, USA, ${ }^{5}$ Department of Ophthalmology, Tufts University of Medicine, Boston, MA, USA and ${ }^{6}$ University of Rochester Eye Institute, University of Rochester Medical Center, Rochester, NY, USA

Email: Gareth R Howell - gareth.howell@jax.org; Richard T Libby - Richard_Libby@URMC.Rochester.edu; Jeffrey K Marchant - jeffrey.marchant@tufts.edu; Lawriston A Wilson - larry.wilson@jax.org; Ioan M Cosma - mihai.cosma@jax.org; Richard S Smith - richard.smith@jax.org; Michael G Anderson - michael-g-anderson@uiowa.edu; Simon WM John* - simon.john@jax.org

* Corresponding author
\end{abstract}

Published: 3 July 2007

BMC Genetics 2007, 8:45 doi:10.1 186/147I-2156-8-45
Received: 9 January 2007

Accepted: 3 July 2007

This article is available from: http://www.biomedcentral.com/I47I-2/56/8/45

(c) 2007 Howell et al; licensee BioMed Central Ltd.

This is an Open Access article distributed under the terms of the Creative Commons Attribution License (http://creativecommons.org/licenses/by/2.0), which permits unrestricted use, distribution, and reproduction in any medium, provided the original work is properly cited.

\begin{abstract}
Background: The glaucomas are a common but incompletely understood group of diseases. DBA/ 2] mice develop a pigment liberating iris disease that ultimately causes elevated intraocular pressure (IOP) and glaucoma. We have shown previously that mutations in two genes, Gpnmb and Tyrp I, initiate the iris disease. However, mechanisms involved in the subsequent IOP elevation and optic nerve degeneration remain unclear.
\end{abstract}

Results: Here we present new mouse strains with Gpnmb and/or Tyrp I genes of normal function and with a DBA/2J genetic background. These strains do not develop elevated IOP or glaucoma with age.

Conclusion: These strains provide much needed controls for studying pathogenic mechanisms of glaucoma using DBA/2J mice. Given the involvement of Gpnmb and/or Tyrp/ in areas such as immunology and tumor development and progression, these strains are also important in other research fields.

\section{Background}

Glaucomas are heterogeneous diseases that affect 70 million people worldwide [1]. They are characterized by the loss of retinal ganglion cells and degeneration of the optic nerve $[2,3]$. Glaucoma is often associated with an agerelated elevation of intraocular pressure (IOP) [4]. Elevated IOP induces glaucomatous neurodegeneration in susceptible individuals. IOP elevation involves compro- mised drainage of aqueous humor $(\mathrm{AqH})$ through the ocular drainage structures in the iridocorneal angle (angle) [5]. Nevertheless, the molecular etiology of IOP elevation varies between individuals and with the type of glaucoma. In pigmentary glaucoma, the iris is damaged resulting in dispersal of iris pigment and debris into the anterior chamber and AqH drainage structures. In susceptible individuals, the pigment and/or debris induces a 
process(es) that damages the drainage structures resulting in high IOP and subsequent glaucoma [6-8]. DBA/2J (D2) mice inherit a disease with similarities to human pigmentary glaucoma $[9,10]$, including ultrastructurally abnormal melanosomes [11]. Around 5-6 months of age, D2 mice develop a pigment dispersing iris disease. Pigment and/or iris debris induces drainage structure damage that disrupts the outflow of aqueous humor. This causes an elevation of IOP that first occurs in a significant portion of mice around 8-9 months of age [10]. In our colony, about $70 \%$ of D2 mice develop moderate or severe glaucoma by 12 months of age [10]. There are now a number of independent reports on DBA/2 glaucoma including several recent ones (e.g. [10,12-14]). Some but not all of these reports suggest earlier onset damage than we have reported. This earlier onset damage clearly does not occur in our colony as we have studied large numbers of mice [10]. We have previously discussed possible explanations for these differences including the exact substrain used and environmental differences.

We have previously shown that the $\mathrm{D} 2$ iris disease is genetically separable into two distinct traits, iris pigment dispersion (IPD) and iris stromal atrophy (ISA) [15]. IPD is characterized by a breakdown of the posterior iris pigment epithelium, slit-like transillumination and prominent pigment dispersion. IPD is caused by a mutation in the glycoprotein (transmembrane) $\mathrm{nmb}$ gene $\left(G p n m b^{R 150 X}\right)$, a recent mutation that arose on D2 in the early 1980 's $[10,16]$. ISA is characterized by deterioration of the iris stroma, and an accumulation of stromal pigment and cell debris in the drainage structures [15], and is caused by a recessive mutation in the tyrosinase related protein 1 gene $\left(\right.$ Tyrp $\left.1^{b}\right)[16]$. D2 mice are homozygous for both GpnmbR150X and Tyrp $1^{\mathrm{b}}$ mutations and inherit a much more severe iris disease and subsequent glaucoma than mice carrying either of the two mutations alone [16]. The published characterization of both IPD and ISA was necessarily performed using mice of a mixed genetic background when mapping and identifying the genes that underlie these traits. These phenotypes have never been fully analyzed in the context of a D2 genetic background.

D2 is one of the most widely used mouse strains in glaucoma research. Here, we describe the development of novel strains that can be used in conjunction with $\mathrm{D} 2$ to further our understanding of the complex mechanisms involved in pigment dispersing iris disease, IOP elevation and glaucoma. Prior to developing these strains, there were no control, age and strain-matched DBA/2J mice that did not develop these phenotypes. Control D2.B6Tyrp $1^{B 6} \mathrm{Gpnmb} b^{B 6} / \mathrm{Sj}$ mice (hereafter referred to as D2.Tyrp $1^{B 6} \mathrm{Gpnmb}^{B 6}$ ) are homozygous for wild type alleles of Tyrp1 and Gpnmb that are derived from strain C57BL/6J (B6). These mice do not develop any iris disease, IOP ele- vation or glaucoma. DBA/2J-Gpnmb $/$ Sj mice (hereafter referred to as D2-Gpnmb ${ }^{+}$) are homozygous for a wild-type allele of Gpnmb on a D2 genetic background. This Gpnmb ${ }^{+}$ allele is the original D2 allele that predates the R150X mutation (See Methods). These mice develop a very mild form of iris disease due to the Tyrp $1^{b}$ mutation, but do not develop elevated IOP or glaucoma. These strains further define the relative contributions of the Tyrp1 and Gpnmb genes to the $\mathrm{D} 2$ phenotype and will serve as powerful control strains in future studies of glaucoma.

\section{Results}

We have developed control strains that will maximize the potential of D2 mice in understanding the pathogenesis of both pigment dispersing iris disease and glaucoma. For

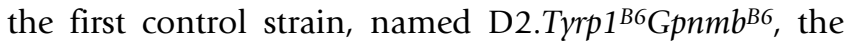
mutated forms of Tyrp1 and Gpnmb were replaced with wild-type alleles from B6 (Tyrp $1^{B G}$ and $G p n m b^{B G}$, see Methods). Mutations in Tyrp1 alter coat color in various strains [17]. As a consequence of the Tyrp1 ${ }^{B G}$ allele, D2.Tyrp $1^{B 6} \mathrm{G} p n m b^{B 6}$ mice have a darker coat color than D2 mice (Figure 1a). The extent of B6-derived sequence flanking both Tyrp1 and Gpnmb genes in this strain was assessed using polymorphic markers. For Tyrp $1^{B 6}$, the proximal breakpoint was mapped to between D4Mit151 and D4Mit1 78 and the distal breakpoint to between D4Mit185 and D4Mit146 (Figure 1b). The maximum size of the B6derived region flanking Tyrp $1^{B 6}$ is $50 \mathrm{Mb}$ or $1.9 \%$ of the mouse genome. For Gpnmb ${ }^{B 6}$, the proximal breakpoint was mapped to between D6Mit268 and D6Mit207, and the distal breakpoint to between D6Mit277 and D6Mit16 (Figure 1c). The maximum amount of $\mathrm{B} 6$ sequence flanking $G p n m b^{B G}$ is estimated to be $36 \mathrm{Mb}$ or $1.4 \%$ of the mouse genome.

\section{D2.Tyrp I $^{\mathrm{B}} \mathrm{GPpmb}^{\mathrm{B} 6}$ show no iris disease, IOP elevation or glaucoma}

As previously reported, the irides of D2 mice with both the GpnmbR150X and Tyrp $1^{b}$ mutations develop severe pigment dispersion and iris atrophy (Figure 2a-c). However, as expected, the irides of D2.Tyrp1 ${ }^{B 6} G p n m b^{B 6}$ mice appear totally normal at all ages. IOP elevation is a key risk factor for glaucoma and occurs in D2 mice as a result of the iris disease. Typically, a population of D2 mice shows elevated IOP levels that peak at around 9-10 months of age (Figure 3a). In contrast, IOPs of D2.Tyrp $1^{B 6} \mathrm{G} p n m b^{B 6}$ mice are not elevated compared to young mice at any assessed age (Figure $3 \mathrm{~b}$ ).

D2.Tyrp $1^{B 6} G p n m b^{B 6}$ mice were further assessed for optic nerve damage. The amount of RGC axon damage was determined using a three point damage rating system based on the amount of axon damage detected in the retrorbital portion of the optic nerve [10]. This system has been validated against axon counting $[18,19] .70 \%$ of D2 

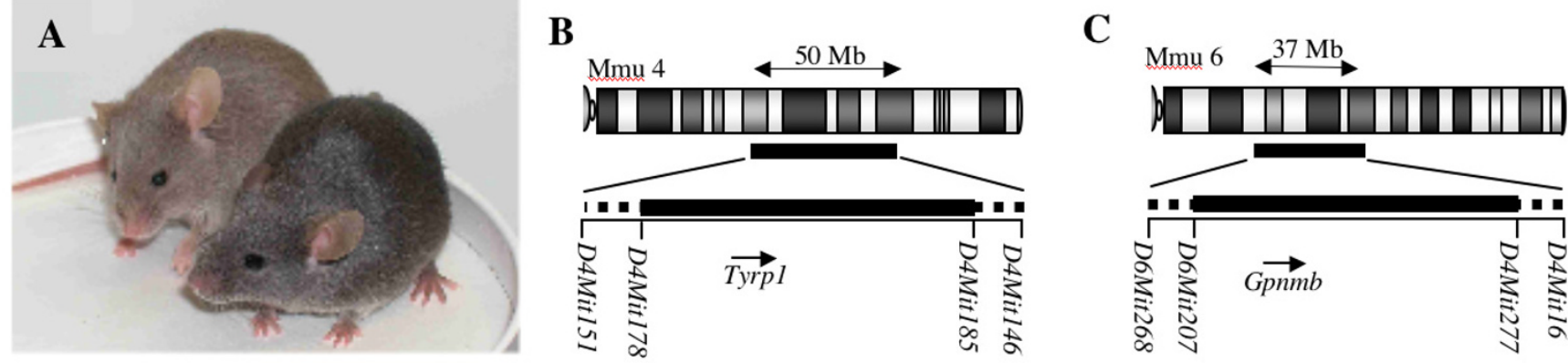

\section{Figure I}

Analysis of D2.Tyrp ${ }^{B 6} G p n m b^{B 6}$ mice. A. D2.Tyrp / ${ }^{B 6} G p n m b^{B 6}$ mice (right) show a darker coat color compared to D2 mice (left) due to the presence of a wild-type Tyrp/ gene. B-C. Regions of B6-derived sequence containing Tyrpl (B) and Gpnmb (C) are indicated by the black horizontal lines, dotted lines indicate the region within which the breakpoints lie. The estimated maximum sizes of the regions (taken from Ensembl) are indicated. Polymorphic markers flanking the breakpoint regions are shown and the orientation of the genes are indicated below.

mice have glaucomatous optic nerve damage by 12 months of age (Figure 3c). In contrast, D2.Tyrp1 $1^{B 6} \mathrm{G} p n m b^{B 6}$ show no evidence of glaucomatous damage at 12 months of age (Figure $3 \mathrm{~d}$ ).

D2.Tyrp1 $1^{B 6} G p n m b^{B 6}$ mice have B6-derived chromosomal regions (congenic intervals) flanking Tyrp1 and Gpnmb respectively. Together, these B6-derived regions represent up to $3.3 \%$ of the genome (Figure 1). To produce an even more closely matched control strain, we developed D2Gpnmb ${ }^{+}$, a second control strain that has no B6-derived congenic intervals. To generate the D2-Gpnmb ${ }^{+}$strain, the Gpnmb ${ }^{R 150 X}$ allele of modern day D2 mice was replaced with the Gpnmb $b^{+}$allele from the D2-sdy strain (the original wild-type D2 Gpnmb allele, see Methods).

\section{D2-Gpnmb ${ }^{+}$mice develop a mild iris disease}

D2-Gpnmb $b^{+}$mice were aged and assessed for ISA and IPD. Although wild-type for Gpnmb, D2-Gpnmb ${ }^{+}$mice are homozygous for the Tyrp $1^{b}$ mutation. Therefore, they are expected to develop the ISA iris disease that is caused by this mutation. A mild ISA disease is observed in these mice (Figure $4 \mathrm{a}-\mathrm{c}$ ). The ISA phenotype in D2-Gpnmb ${ }^{+}$ mice is characterized by a change in iris stromal morphology (appearing "roughened" and lacking complexity). However, the iris shows no evidence of IPD, maintains integrity, and is overall very mildly affected when compared to the severe, combined ISA and IPD phenotype of D2 mice.

\section{D2-Gpnmb ${ }^{+}$mice do not develop elevated IOP or glaucoma}

IOP was assessed at a variety of ages between 4 months and 23 months of age. The selected ages are relevant to the window of IOP changes observed in DBA/2J mice. There was no age-related increase of IOP in these mice. Additionally, no D2-Gpnmb ${ }^{+}$mice had IOP values above 21 $\mathrm{mmHg}$, levels considered to be glaucoma-suspect in people (Figure 4d). Consistent with this, we detected no signs of glaucomatous RGC death and optic nerve degeneration in D2-Gpnmb ${ }^{+}$mice. D2 mice show RGC death, loss of the nerve fiber layer and severe optic nerve cupping by 12 months of age [10]. Retina and optic nerve morphology was examined in 4 eyes taken from D2-Gpnmb ${ }^{+}$mice at each of 4.5 months, 12 months and 18 months of age (Figure $5 \mathrm{a}-\mathrm{c}$ ). The RGC layer and nerve fiber layer was normal, and there was no evidence of optic nerve head cupping (Figure 5d). Optic nerve damage was further assessed by sectioning a portion of the retro-orbital optic nerve and staining with PPD, a stain that sensitively detects degenerating axons. There was no evidence of glaucomatous optic nerve damage in D2-Gpnmb ${ }^{+}$eyes taken from a large number of mice between 4.5 and 15 months of age (Figure 5e-i). Importantly, there was no correlation between optic nerve damage and measured IOP level in individual mice and thus the range of pressure seen in D2-Gpnmb ${ }^{+}$did not affect nerve damage. In addition, we compared axon number in young pre-glaucomatous D2 eyes with aged D2-Gpnmb ${ }^{+}$eyes, using a previously described counting system $[18,19]$, and there was no significant difference in axon number $(51,748 \pm 907$ for D2, and 52,074 \pm 427 for D2-Gpnmb ${ }^{+}, \mathrm{n}=10$ for each group, $\mathrm{p}=0.7)$. A moderate form of optic nerve damage was present in one eye from a 12 months old mouse, and 2 eyes from mice over 16 months of age. This may be a very rare case of glaucoma due to the mutant Tyrp1 gene, or more likely age-related damage that is not related to glaucoma as a similar degree of damage is observed in other non-glaucomatous mouse strains of a similar age $[12,20]$. 

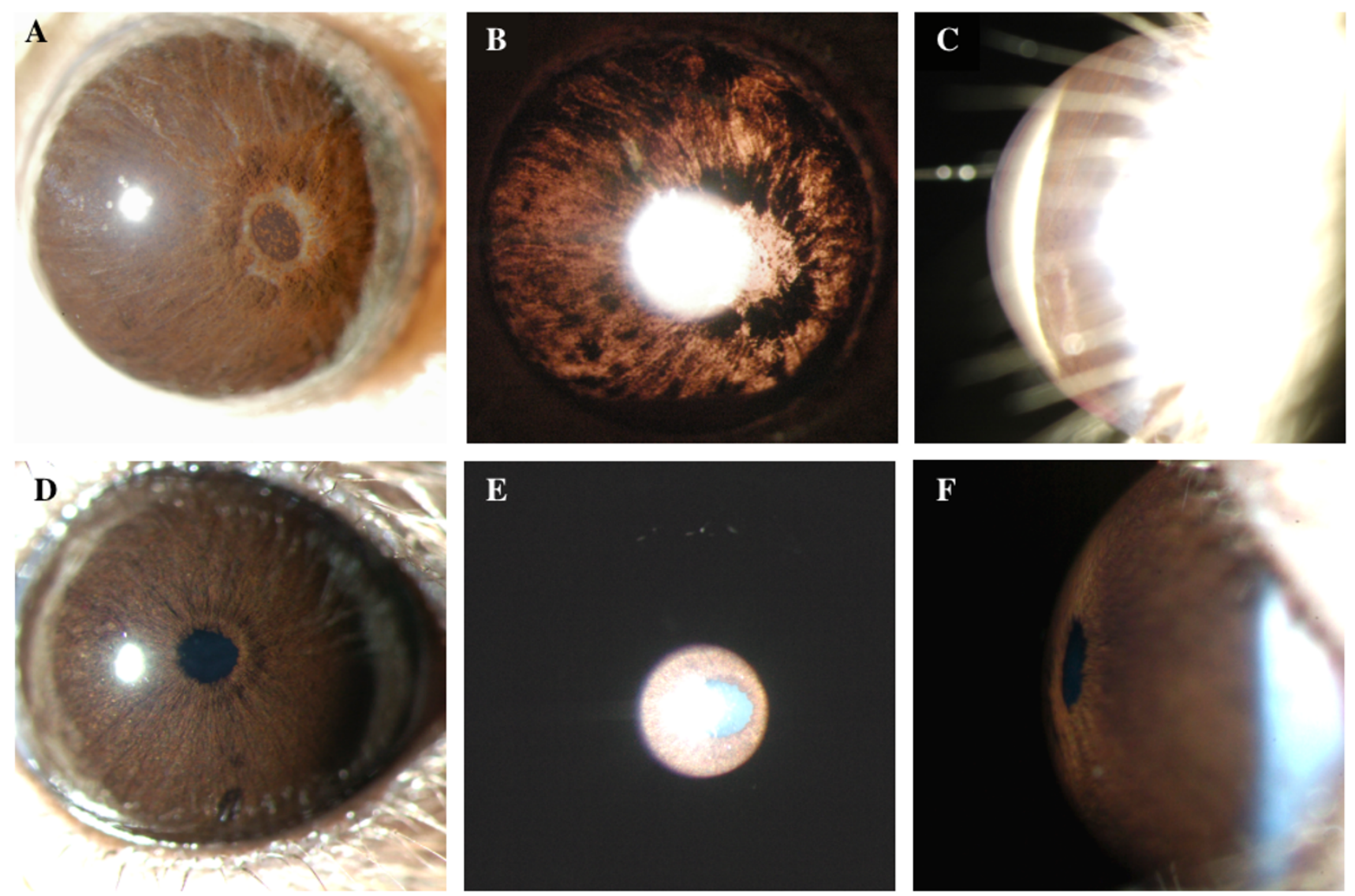

Figure 2

Comparison of iris disease in 13 months old D2 mice and D2.Tyrp/ ${ }^{B 6} G$ Gnmb ${ }^{B 6}$ mice. $\mathbf{A}$ and $\mathbf{D}$ show broad-beam illumination, $\mathbf{B}$ and $\mathbf{E}$ show transillumination defects and $\mathbf{C}$ and $\mathbf{F}$ show the relative dimensions of the anterior chamber. A-C. In D2 mice, the iris disease involves iris stromal atrophy, transillumination defects and progressive depigmentation with abnormal dispersal of iris pigment into the anterior chamber. D-F. In contrast, D2.Tyrp / ${ }^{B 6} G p n m b^{B 6}$ mice show no iris pigment disease, transillumination defects or alteration of the anterior chamber.

\section{Discussion}

DBA/2J is an established mouse model for inherited glaucoma that shows iris pigment dispersion, IOP elevation and optic nerve degeneration. D2 mice are homozygous for both the GpnmbR150X and Tyrp $1^{\mathrm{b}}$ mutations. Both of these mutations are necessary for the severe iris pigment disease that leads to IOP elevation and glaucoma [16]. Although DBA/2J is an experimentally tractable model of glaucoma, it is an inbred mouse strain and so no strain matched DBA/2J controls without glaucoma have been available. Here we describe the generation of two derivative strains of DBA/2J (D2.Tyrp1 $1^{B 6} G p n m b b^{B 6}$ and D2Gpnmb ${ }^{+}$), where either the mutant alleles of both Gpnmb and Tyrp1 or only Gpnmb have been replaced by wild-type versions. D2.Tyrp1 $1^{B 6} \mathrm{Gpnmb} b^{B 6}$ mice contain a maximum of $3.3 \%$ of B6-derived sequence on a D2 background. In the case of D2-Gpnmb ${ }^{+}$, the only known difference compared to D2 is a single base change in the Gpnmb gene. Neither D2.Tyrp1 ${ }^{B 6}{ }^{B} p n m b^{B 6}$ nor D2-Gpnmb ${ }^{+}$strains develop IOP elevation or glaucoma.

Since determining that the Gpnmb mutation recently arose on the DBA/2J background [10], we have produced a further control strain, D2.Tyrp1 ${ }^{B 6} \mathrm{Gpnmb}^{+}$, that is currently being characterized. This strain contains a congenic interval flanking Tyrp1 but is improved over D2.Tyrp $1^{B 6} G p n m b^{B 6}$ in that it lacks a congenic interval around Gpnmb (as the Gpnmb allele is the ancestral DBA/ 2J allele). This strain is wild-type for both glaucoma inducing genes and is not expected to develop any D2 glaucoma-associated phenotypes. Although characterization of large numbers of these mice is a lengthy undertak- 

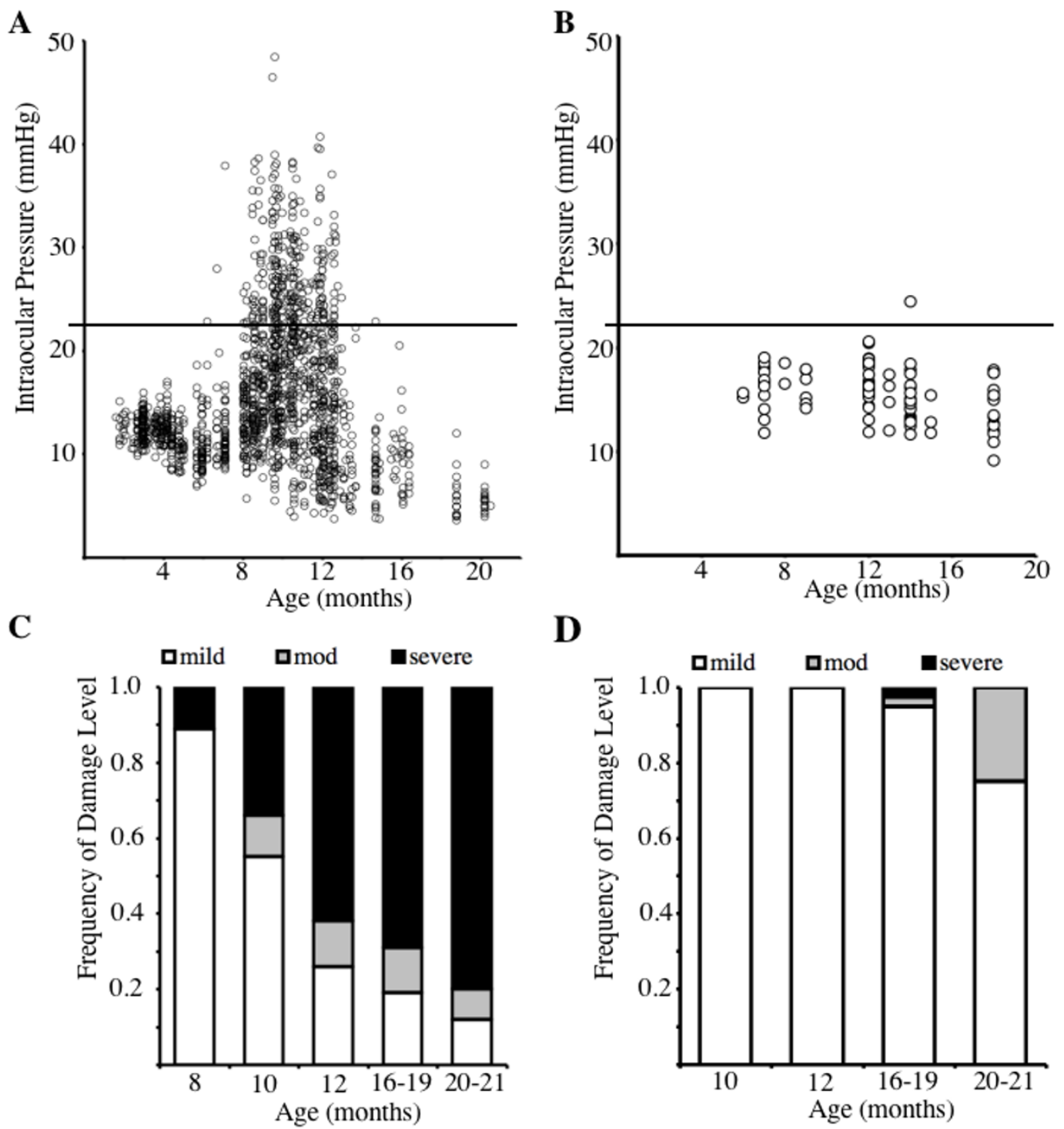

Figure 3

Assessment of IOP levels and glaucoma in D2.Tyrp / ${ }^{B 6} \mathrm{Gpnmb} b^{B 6}$ mice. A. IOP profile of a population of D2 mice (I437 IOPs) between 4 and 20 months of age. B. IOP profile of 88 D2.Tyrp /B6 Gpnmb ${ }^{B 6}$ mice between 6 and I 8 months of age. Individual IOP measurements are plotted as circles. The horizontal line indicates $21 \mathrm{mmHg}$, a value considered to be glaucoma-suspect in people. IOP elevation above $21 \mathrm{mmHg}$ is first observed in D2 mice at around 6 months of age, and peaks at 9-10 months. D2.Tyrp / ${ }^{B 6} G p n m b^{B 6}$ mice show no significant IOP elevation above $21 \mathrm{mmHg}$. C. Previously reported frequencies of optic nerve damage in D2 mice are presented showing clear glaucomatous damage. Approximately $70 \%$ of D2 eyes have moderate or severe damage by 12 months of age, and $80 \%$ by $16-19$ months of age. D. In contrast no glaucomatous damage was seen in 18 D2.Tyrp / ${ }^{B 6} \mathrm{Gpnmb} b^{B 6}$ eyes at 12 months of age, and only 5\% (3 of 39) of eyes from 16-19 months of age had obvious disease. A total of 72 eyes were analyzed for all ages. Mild nerves have fewer than $5 \%$ damaged axons, moderate (mod) nerves have between $5 \%$ and $50 \%$ axon damage, and severe nerves have greater than $50 \%$ axons damaged. Only moderate and severe nerves are considered to show glaucomatous damage, as mild damage is frequently observed in aging non-glaucomatous strains by 10 to 12 months of age. At 12 months of age, moderate damage is very unusual in non-glaucomatous strains and so we consider this degree of damage a sign of glaucoma. Although moderate damage is not common in non-glaucomatous mice, it does occur in more mice of non-glaucomatous strains by 16 to 18 months. Thus, at these older ages, moderate damage may be caused by an unusual degree of age-related RGC demise or by glaucoma. The single case of severe damage is extremely rare in control mice and may or may not represent glaucoma. 

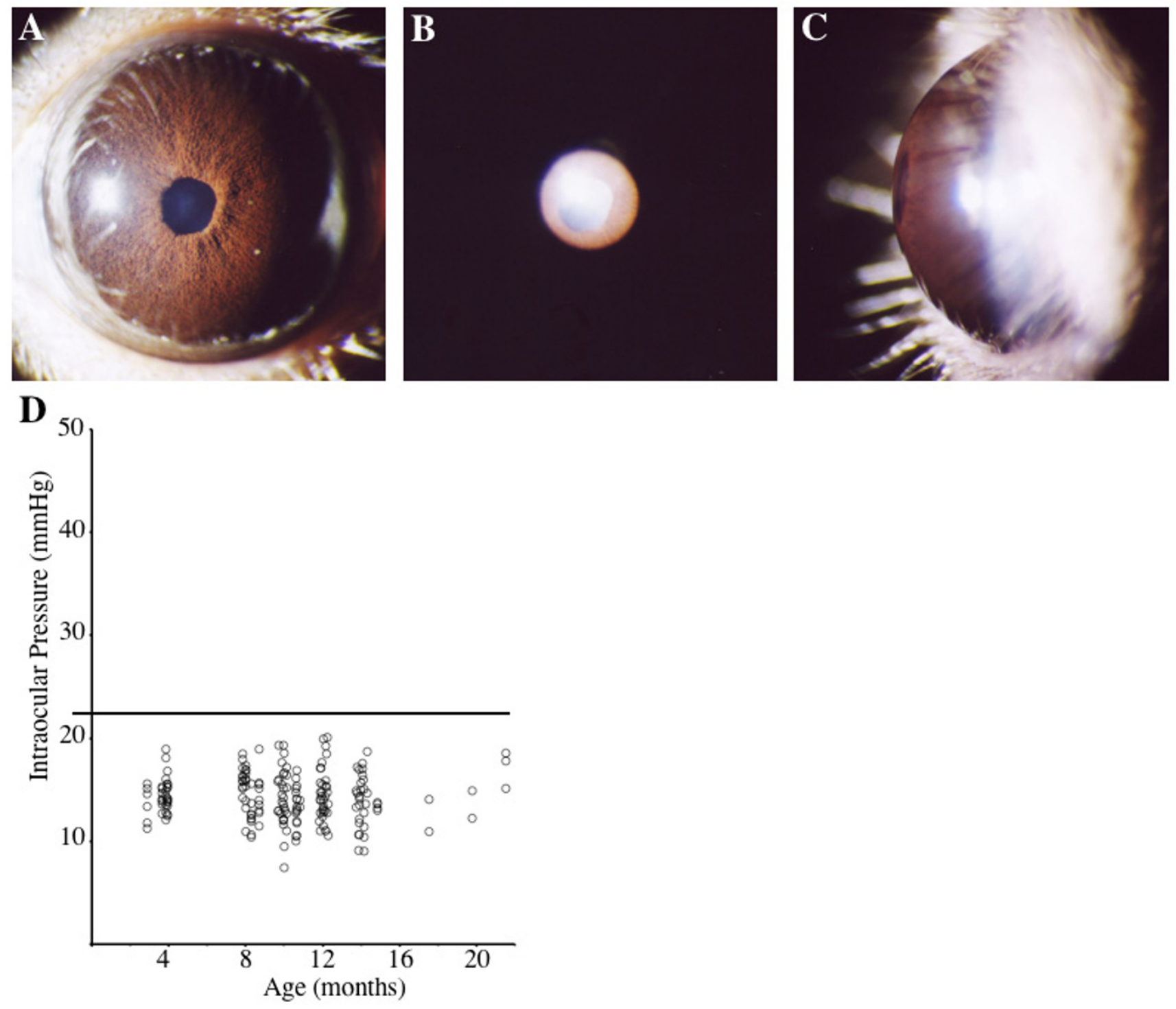

\section{Figure 4}

A-C. Iris disease and IOP in D2-Gpnmb $b^{+}$mice. A. Broad-beam illumination. B. Transillumination. C. Side view dimensions of the anterior chamber. D2-Gpnmb ${ }^{+}$eyes show no IPD but have a distinct form of ISA where the iris appears 'roughened'. D2$G$ pnmb $b^{+}$eyes have no transillumination defects and normal anterior chamber morphology (more than 20 eyes were analyzed from a variety of ages, images shown here are from a 13 months old mouse). D. IOP measurements in D2-Gpnmb $b^{+}$mice. Individual IOP readings are plotted as circles. The solid horizontal line indicates $21 \mathrm{mmHg}$, a value considered to be glaucoma-suspect in people. No 'high' IOP readings were seen in 97 eyes measured. Also, there is no significant change in IOP levels comparing 4 months old D2-Gpnmb ${ }^{+}$mice to the $8,10,12$ or 14 months old groups ( $>0.37$ for all comparisons).

ing, initial analyses agree with the findings reported here for D2.Tyrp $1^{B 6} G p n m b^{B 6}$ mice, with no evidence of iris disease, IOP elevation or glaucoma.

Regarding the control strains, the following points are pertinent. Although they lack all iris disease and glaucoma, the D2.Tyrp1 $1^{B 6} G p n m b^{B 6}$ strain does not typically reproduce as well as the D2-Gpnmb ${ }^{+}$strain and appears to have an increased incidence of early deaths (based on retrospective observations, but no specific spontaneous death study has been conducted). Although not well characterized, the decreased fecundity appears to be related to the Tyrp $1^{\mathrm{B} 6}$ allele on a D2 background, since other D2 strains that we have been producing with this allele have exhibited a similar phenotype. These issues do not prevent the use of this strain. However, since the D2-Gpnmb ${ }^{+}$strain 

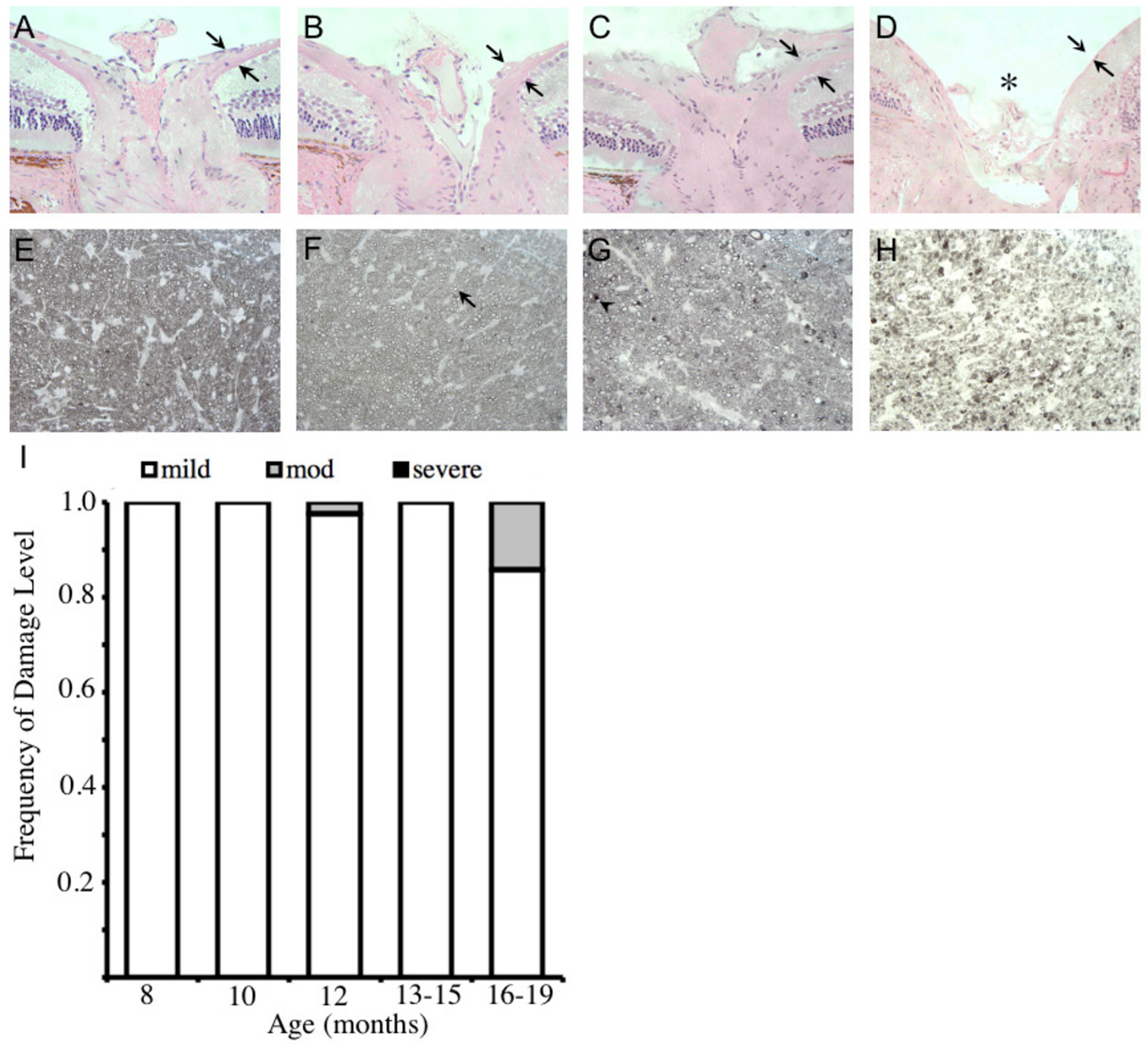

\section{Figure 5}

D2-Gpnmb $b^{+}$mice do not develop glaucoma. A-C. H\&E stained sections of D2-Gpnmb $b^{+}$mice at $4.5 \mathrm{mo}$ (A), $12 \mathrm{mo}$ (B) and 18 mo (C) showing a normal nerve fiber layer (arrows) and no evidence of optic nerve cupping. D. H\&E section of a 12 months old D2 mouse with severe glaucoma, showing optic nerve cupping (asterisk) and loss of nerve fiber layer (arrows). E-G. PPD staining of the retro-orbital optic nerve from a $4.5 \operatorname{mos}(\mathbf{E}), 12 \mathrm{mos}(\mathbf{F}), 18 \mathrm{mos}(\mathbf{G}) \mathrm{D} 2-G p n m b^{+}$mice. These nerves are healthy with only rare degenerating axons, as is typical for nerves of non-glaucomatous mouse strains. $\mathbf{H}$. Severe glaucoma in a 12 mos D2 eye. Healthy axons have clear axoplasm, surrounded by darkly stained myelin (arrow). The sick or dying axons stain grey/black (arrowhead). (I) Frequency of glaucomatous optic nerve damage in D2-Gpnmb ${ }^{+}$mice, using the 3 point grading system (see Methods). No D2-Gpnmb ${ }^{+}$mice show severe glaucoma, and only 3 eyes show moderate damage. Importantly, these moderately affected nerves only just enter this grade (all had less than 10\% axon loss), and were not as severely degenerated as the typical D2 moderate nerve.

lacks these issues and has no B6 derived interval, we recommend it as a control when studying glaucomatous neurodegeneration. Although the D2-Gpnmb $b^{+}$strain still develops a mild iris disease, glaucomatous nerve damage was clearly absent in the vast majority, if not all, of these mice. Thus, despite the spread in IOP, the highest IOPs 
were not significant enough to induce glaucomatous axon loss. When using these control strains, it is important to remember that they may still have some undefined but glaucoma relevant phenotype(s).

It is clear that glaucoma is a multifactorial disease and D2 mice are likely to have susceptibility factors in addition to those associated with the Gpnmb and Tyrp1 genes. Thus, although these strains do not develop glaucoma, as defined by the absence of nerve damage, they may still have other phenotypes that render the D2 background susceptible to glaucoma. Such phenotypes may or may not have contributed to nerve the damage detected in a small percentage of mice.

Tyrp1 and Gpnmb function in melanosomes, organelles that produce pigment. Manifestation of both IPD and ISA in $\mathrm{D} 2$ eyes is dependent upon active pigment production $[16,20]$. The mechanisms involved in IPD and ISA and the subsequent IOP elevation are complex and in addition to pigment production appear to involve immunity $[21,22]$. Ongoing functional mouse genetics experiments complemented with a variety of immunological, molecular and cellular techniques are vital to unravel these mechanisms. The control strains described here will be a valuable resource in elucidating the biological mechanisms involved in pigment-related iris disease. In addition to ocular-related diseases, these strains provide an important resource to test the roles of Tyrp1 and Gpnmb in other diseases. Tyrp1 is the most common melanoma antigen and Gpnmb is used as a marker for metastatic melanomas [23] and has recently been identified as a potential molecular therapeutic target in patients with glioblastoma multiforme [24]. Gpnmb may also be important in antigen presenting cells that control immune responses [21].

To improve our understanding of glaucoma, it is essential that genes and pathways involved in IOP elevation and glaucomatous RGC and optic nerve degeneration are identified. By analyzing gene expression profiles in D2 mice at various ages [25], and conducting subsequent functional tests, it is possible to identify differentially expressed genes important for the onset and/or progression of glaucoma. Individual mouse strains, such as D2, have a unique collection of alleles affecting many biological processes and that affect gene expression levels and protein activity [26]. Therefore, many gene expression differences are observed between D2 and other naturally occurring strains that do not get glaucoma [27]. This can complicate experiments and make it difficult to prioritize genes for subsequent functional testing. Additionally, it is possible that strain differences can mask some expression changes that occur in D2 mice and are relevant to the glaucoma. By enabling analysis of age- and strain-matched control mice, the control strains reported here alleviate the difficulties of controlling experiments using D2 mice.

\section{Conclusion}

The strains describes here will enable the systematic evaluation of the processes involved in iris disease, IOP elevation and glaucoma, work that is likely to provide novel understanding and lead to targets for improved therapeutics for human glaucomas.

\section{Methods}

\section{Animal husbandry and strain development}

Mice were housed in a $14 \mathrm{~h}$ light to $10 \mathrm{~h}$ dark cycle under previously described conditions $[9,15]$. The Jackson Laboratory's (Bar Harbor, Maine, United States) pathogen surveillance program regularly screened for pathogens. All experiments were conducted in accordance with the Association for Research in Vision and Ophthalmology's statement on the use of animals in ophthalmic research and were approved by our institutional animal care and use committee. Modern DBA/2J (D2) mice (\#000671, see [28]) have mutations in both Tyrp1 and Gpnmb. Although the majority of included data for modern D2 mice has been previously published, over 300 modern D2 mice were aged and analyzed over the same period of time as the mice of the other strains presented here. For the generation of the D2.Tyrp1 ${ }^{B 6}{ }^{6} p n m b^{B 6}$ strain, D2 mice were crossed to C57BL/6J (B6) to create F1s. Progeny carrying the B6 allele of both Gpnmb (Gpnmb $\left.{ }^{B 6}\right)$ and Tyrp1 $\left(\right.$ Tyrp $\left.^{B G}\right)$ were then backcrossed to $\mathrm{D} 2$ for ten generations. Brother/sister matings were then established to generate mice homozygous for Gpnmb $b^{B 6}$ and Tyrp1 $1^{B 6}$ and to maintain a stable D2.Tyrp1 ${ }^{B 6} \mathrm{G} p n m b^{B 6}$ doubly homozygous colony.

The Gpnmb R150X mutation arose in the early 1980s and became fixed in the ancestors of modern day D2 mice [16]. The $s d y$ mutation alters coat color and occurred in DBA/2J (D2) mice in 1983. At that point, the DBA/2J$D t n b p 1^{s d y}$ strain (hereafter referred to as D2-sdy) was separated from the main D2 colony. Genotyping a D2-sdy colony for Gpnmb in the early 2000s revealed that these mice had an original wild-type allele of Gpnmb $\left(G p n m b^{+}\right)$. To develop the D2-Gpnmb ${ }^{+}$strain, D2-sdy were crossed to modern D2 mice for three generations. We are continuing to backcross D2-Gpnmb ${ }^{+}$to modern day D2 to further reduce the possibility of $\mathrm{D} 2-\mathrm{G} n n m b^{+}$mice harboring unknown genetic differences compared to modern day $\mathrm{D} 2$ mice. These higher generation $\mathrm{D} 2-\mathrm{G} p n m \mathrm{~b}^{+}$mice will be provided to the community. (They are being accepted for distribution by Jackson Laboratory mouse resources and will become strain DBA/2J-Gpnmb ${ }^{+} / \mathrm{SjJ}$ with stock \# 007048). However, to hasten characterization of mice with a D2 genetic background and a wild type allele of Gpnmb, brother/sister matings that did not carry the $s d y$ 
mutation were selected to establish the D2-Gpnmb+ strain that is homozygous for the wild-type Gpnmb allele and characterized here. These matings also produced the mice for clinical examinations, IOP measurements and assessment of glaucomatous damage. Analysis of 102 microsatellite markers (average spacing $13.25 \mathrm{cM}$ ) revealed no allelic differences between D2-Gpnmb ${ }^{+}$and modern day D2.

\section{Genotyping}

The GpnmbR150X mutation creates a novel PvuII enzyme site [16]. DNA isolated from tail snips was used to PCR amplify the 125 bp region surrounding the mutation using primers nmb7 (CTACAACTGGACTGCAGGGG) and nmb8 (AGCTCCATTTCTTCCATCCA). The resulting product was digested with $P v u I I$ (NEB). The presence of the mutation is indicated by the presence of two bands of $50 \mathrm{bp}$ and $75 \mathrm{bp}$. Identification of B6-derived sequence that included Tyrp1 on Chromosome 4 was achieved using the flanking polymorphic markers D4Mit178 (D2 = $170 \mathrm{bp}, \mathrm{B} 6=146 \mathrm{bp})$ and D4Mit327 (D2 = $92 \mathrm{bp}, \mathrm{B} 6=$ $106 \mathrm{bp})$. Identification of B6-derived sequence that included Gpnmb on Chromosome 6 was achieved using the flanking polymorphic markers D6Mit74 (D2 $=150$ bp, B6 = $160 \mathrm{bp}$ ) and D6Mit355 (D2 = $106 \mathrm{bp}, \mathrm{B} 6=124$ bp). The extent of the B6-derived region was analyzed using polymorphic markers spanning Chromosomes 4 and 6 (see [29]).

\section{Clinical examinations}

We examined eyes at 2-24 months of age with a slit-lamp biomicroscope (Haag-Streit) and photographed them with a $40 \times$ objective lens. Phenotypic assessment of iris stromal atrophy, dispersed pigment and transillumination was carried out as described previously $[9,15,20]$.

\section{IOP and optic nerve assessment}

Mice were acclimatized to the procedure room, anesthetized by using an intraperitoneal injection of ketamine/ xylazine mixture and IOP measured as previously described [30]. Optic nerve cross sections were examined for glaucomatous damage using a modified paraphenylenediamine (PPD) staining protocol to stain the myelin sheath of all axons and the axoplasma of damaged axons as described previously [18]. Optic nerves were assessed for glaucomatous damage using a non-biased three point grading system that has been validated against axon counting $[18,19]$. Axon counts were carried out as previously described $[18,19] .10$ randomly selected D2 eyes (pre-glaucomatous, 3 to 4 months) and 10 randomly selected D2-Gpnmb+ eyes (no glaucoma, 10.5 to $11 \mathrm{mos}$ ) were counted.

\section{Histology}

Eyes were fixed in $4 \%$ paraformaldehyde (PFA) overnight at $4{ }^{\circ} \mathrm{C}$ and stored in $0.4 \%$ PFA at $4{ }^{\circ} \mathrm{C}$. For general morphology, eyes were processed, embedded in plastic, sectioned and stained with Hemotoxylin and Eosin.

\section{Authors' contributions}

GH carried out optic nerve assessment of strains, coordinated data collection and analysis and prepared the manuscript. RL participated in the design of the experiment, carried out optic nerve assessment of strains and helped write the manuscript. JKM performed the axon counts. LW organized the generation of the strains, IOP measurements and harvesting. IC carried out the IOP measurements. RS carried out optic nerve assessment. MA participated in the design of the experiment and strain generation, carried out clinical assessments of the mice, and helped write the manuscript. SJ conceived the study and participated in its design and coordination and oversaw all components including manuscript preparation. All authors read and approved the final manuscript.

\section{Acknowledgements}

The authors thank Amy Snow for strain development and maintenance, and IOP measurements. We thank Greg Peterson and the John Lab for technical assistance. We also thank The Jackson Laboratory Scientific Services including Pete Finger and Barbara Mortimer for technical assistance. Scientific support services at The Jackson Laboratory are subsidized by a core grant from the National Cancer Institute (CA34 I96). This work was supported in part by the National Eye institute, EYI I72I (S.W.M.J), F32EYOI45I5 (R.T.L.) and F32EY070I5 (M.G.A.). S.W.M. John is an Investigator of The Howard Hughes Medical Institute.

\section{References}

I. Quigley HA: Number of people with glaucoma worldwide. The British journal of ophthalmology 1996, 80(5):389-393.

2. Hitchings RA: Glaucoma - Fundamentals of Clinical Ophthalmology. London: BMJ Publishing Group; 2000.

3. Ritch R, Shields MB, Krupin T: The Glaucomas: Clinical Science. 2nd edition. St Louis, MO: Mosby-Year Book; 1996.

4. Palmberg PF, Wiggs JL: Mechanisms of Glaucoma. In Ophthalmology 2nd edition. Edited by: Yanoff M, Duker JS. St Louis, MO.: Mosby; 2004.

5. Lutjen-Drecoll E, Rohen JW: Morphology of aqueous outflow pathways in normal and glaucomatous eyes. In The Glaucomas Edited by: Ritch R, Shields MB, Krupin T. St Louis, MO: Mosby-Year Book; 1996:89-123.

6. Campbell DG, Schertzer RM: Pigmentary Glaucoma. In The Glaucomas Edited by: Ritch R, Shields MB, Krupin T. St Louis: Mosby; 1996:975-991.

7. Ritch R: A unification hypothesis of pigment dispersion syndrome. Transactions of the American Ophthalmological Society 1996, 94:38I-405. discussion 405-389.

8. Shields MB: Glaucomas associated with disorders of the iris. In Textbook of Glaucoma 4th edition. Baltimore: Williams and Wilkins; 1992:276-286.

9. John SW, Smith RS, Savinova OV, Hawes NL, Chang B, Turnbull D, Davisson M, Roderick TH, Heckenlively JR: Essential iris atrophy, pigment dispersion, and glaucoma in DBA/2J mice. Investigative ophthalmology \& visual science 1998, 39(6):951-962.

10. Libby RT, Anderson MG, Pang IH, Robinson ZH, Savinova OV, Cosma IM, Snow A, Wilson LA, Smith RS, Clark AF, et al.: Inherited glaucoma in DBA/2J mice: pertinent disease features for studying the neurodegeneration. Vis Neurosci 2005, 22(5):637-648. 
11. Libby RT, Gould DB, Anderson MG, John SW: Complex genetics of glaucoma susceptibility. Annu Rev Genomics Hum Genet 2005, 6:15-44.

12. Danias J, Lee KC, Zamora MF, Chen B, Shen F, Filippopoulos T, Su Y, Goldblum D, Podos SM, Mittag T: Quantitative analysis of retinal ganglion cell (RGC) loss in aging DBA/2NNia glaucomatous mice: comparison with RGC loss in aging C57/BL6 mice. Investigative ophthalmology \& visual science 2003, 44(1 2):5I5I-5I62.

13. Schlamp CL, Li Y, Dietz JA, Janssen KT, Nickells RW: Progressive ganglion cell loss and optic nerve degeneration in DBA/2J mice is variable and asymmetric. $B M C$ neuroscience 2006, 7:66.

14. Schuettauf F, Rejdak R, Walski M, Frontczak-Baniewicz M, Voelker M, Blatsios G, Shinoda K, Zagorski Z, Zrenner E, Grieb P: Retinal neurodegeneration in the DBA/2J mouse-a model for ocular hypertension. Acta neuropathologica 2004, 107(4):352-358.

15. Chang B, Smith RS, Hawes NL, Anderson MG, Zabaleta A, Savinova O, Roderick TH, Heckenlively JR, Davisson MT, John SW: Interacting loci cause severe iris atrophy and glaucoma in DBA/2J mice. Nature genetics 1999, 21 (4):405-409.

16. Anderson MG, Smith RS, Hawes NL, Zabaleta A, Chang B, Wiggs JL, John SW: Mutations in genes encoding melanosomal proteins cause pigmentary glaucoma in DBA/2J mice. Nature genetics 2002, 30(I):8I-85.

17. Zdarsky E, Favor J, Jackson IJ: The molecular basis of brown, an old mouse mutation, and of an induced revertant to wild type. Genetics 1990, 126(2):443-449.

18. Anderson MG, Libby RT, Gould DB, Smith RS, John SW: High-dose radiation with bone marrow transfer prevents neurodegeneration in an inherited glaucoma. Proceedings of the National Academy of Sciences of the United States of America 2005, I 02(I 2):4566-457|.

19. Libby RT, Li Y, Savinova OV, Barter J, Smith RS, Nickells RW, John SW: Susceptibility to neurodegeneration in a glaucoma is modified by Bax gene dosage. PLoS Genet 2005, I(I): 17-26.

20. Anderson MG, Libby RT, Mao M, Cosma IM, Wilson LA, Smith RS, John SW: Genetic context determines susceptibility to intraocular pressure elevation in a mouse pigmentary glaucoma. BMC biology 2006, 4:20.

21. Mo JS, Anderson MG, Gregory M, Smith RS, Savinova OV, Serreze DV, Ksander BR, Streilein JW, John SW: By altering ocular immune privilege, bone marrow-derived cells pathogenically contribute to DBA/2J pigmentary glaucoma. J Exp Med 2003 197(10): I335-1344.

22. Zhou X, Li F, Kong L, Tomita H, Li C, Cao W: Involvement of inflammation, degradation, and apoptosis in a mouse model of glaucoma. The Journal of biological chemistry 2005, 280(35):3|240-3| 248.

23. Weterman MA, Ajubi N, van Dinter IM, Degen WG, van Muijen GN, Ruitter DJ, Bloemers HP: nmb, a novel gene, is expressed in lowmetastatic human melanoma cell lines and xenografts. International journal of cancer 1995, 60(I):73-8I.

24. Kuan CT, Wakiya K, Dowell JM, Herndon JE 2nd, Reardon DA, Graner MW, Riggins G], Wikstrand C], Bigner DD: Glycoprotein nonmetastatic melanoma protein $B$, a potential molecular therapeutic target in patients with glioblastoma multiforme. Clin Cancer Res 2006, I 2(7 Pt I): 1970-1982.

25. Steele MR, Inman DM, Calkins DJ, Horner PJ, Vetter ML: Microarray analysis of retinal gene expression in the DBA/2J model of glaucoma. Investigative ophthalmology \& visual science 2006, 47(3):977-985.

26. Wade CM, Daly MJ: Genetic variation in laboratory mice. Nature genetics 2005, 37(I I): I I75-I I 80.

27. Nadler JJ, Zou F, Huang H, Moy SS, Lauder J, Crawley JN, Threadgill DW, Wright FA, Magnuson TR: Large-scale gene expression differences across brain regions and inbred strains correlate with a behavioral phenotype. Genetics 2006, 174(3): 1229-1236.

28. Jaxmice [http://jaxmice.jax.org]

29. Mouse Genome Informatics [http://www.informatics.jax.org]

30. Savinova OV, Sugiyama F, Martin JE, Tomarev SI, Paigen B], Smith RS John SW: Intraocular pressure in genetically distinct mice: an update and strain survey. BMC Genet 200I, 2:12.
Publish with Bio Med Central and every scientist can read your work free of charge

"BioMed Central will be the most significant development for disseminating the results of biomedical research in our lifetime. "

Sir Paul Nurse, Cancer Research UK

Your research papers will be:

- available free of charge to the entire biomedical community

- peer reviewed and published immediately upon acceptance

- cited in PubMed and archived on PubMed Central

- yours - you keep the copyright

Submit your manuscript here:

http://www.biomedcentral.com/info/publishing_adv.asp
BioMedcentral 